\title{
Bilateral laparoscopic ureterolysis using hydrodissection in retroperitoneal fibrosis: a new application of an old technique
}

This article was published in the following Dove Press journal: Research and Reports in Urology

\author{
Maher Abdessater ${ }^{1}$ \\ Sandra Elias ${ }^{2}$ \\ Johnny Boustany ${ }^{3}$ \\ Raghid El Khoury ${ }^{3}$ \\ 'Urology Department, Centre \\ Hospitalier Régional René DUBOS, \\ Pontoise, 95300, France; ${ }^{2}$ Anesthesia \\ Department, Institut Gustave Roussy, \\ Villejuif, 94550, France; ${ }^{3}$ Urology \\ Department, Notre Dame Des Secours \\ University Medical Center, Byblos, \\ Lebanon
}

Objective: To describe our new laparoscopic approach in ureterolysis using the $\mathrm{ERBEJET}^{\circledR} 2$ device to hydrodissect the ureters in idiopathic retroperitoneal fibrosis.

Methods: Bilateral laparoscopic ureterolysis was done after medical therapy failure (steroids) in a 47 year old patient with retroperitoneal fibrosis. Ureteral stents were placed before surgery. Only five trocars were used for both sides, Toldt's fascia was opened and the dissection was performed till the psoas muscle. The ureters were completely freed from the fibrotic tissue using the ERBEJET 2 device (pure hydrodissection). An omental wrap was passed behind the colonic flexure at the right and placed around the ureter. At the left, we closed Toldt's fascia by running sutures back to the freed ureter.

Results: The operating time was 198 mins. The blood loss was $50 \mathrm{cc}$. The hospital stay was 3 days. The double $\mathrm{J}$ stents were removed at 4 weeks postoperatively. After 2 years, the patient is free of symptoms with normal creatinine level and no urinary tract obstructions.

Conclusions: Hydrodissection of the ureter in retroperitoneal fibrosis is a new application in the upper urinary tract laparoscopic approach that has not been described in literature before. It seems to offer excellent midterm outcomes. The use of ERBEJET 2 device seems to simplify the procedure and make it feasible and safe (less trauma to the ureter and more conservation of its blood supply). We believe that comparative studies are needed to assess the role of hydrodissection in ureterolysis for retroperitoneal fibrosis.

Keywords: hydrodissection, laparoscopic ureterolysis, retroperitoneal fibrosis, new technique

\section{Introduction}

The use of water stream for the surgical dissection of the human tissues have been used in many medical and surgical specialties. ${ }^{1-3,7}$

The hydro surgery has been improving in the previous years, and hydro-jet generators and delivery probes that provide more range of pressure control and delivery accuracy have been developed and used in many studies that showed different effects on tissue planes at varying pressure levels of hydrodissection in human and animal models. ${ }^{4,5}$ For example, pressure settings in the 225-305 psi (16-21 bar) range can be safely utilized with nerve and microvascular preservation. ${ }^{3,5,6}$

In urology, Shekarriz et al, performed the first interventions using the hydrodissection technique for retroperitoneal lymph-node dissection, laparoscopic nephrectomy, and laparoscopic partial nephrectomy (with vascular clamping). ${ }^{5,6,8}$
Correspondence: Maher Abdessater Urology Department, Centre Hospitalier Régional René DUBOS, 5 Quai Bucherelle, 95300 Pontoise, France $\mathrm{Tel}+33787982484$

Email Maher.abdessater@gmail.com 
Moinzadeh et al, did a pioneering work using hydrodissection in a calf model for laparoscopic partial nephrectomy without the need for renal hilar clamping. ${ }^{9}$

In addition, this technique has been used in nerve sparing radical prostatectomy in both open and robotic procedures. $^{10,11}$

Most of these teams used the ERBEJET ${ }^{\circledR} 2$ or Helix HydroJet $^{\mathrm{TM}}$ devices for tissue dissection. Small vessels are usually preserved during hydrodissection as reflected in vivo. ${ }^{3,5,6}$

In a radical prostatectomy, ERBEJET 2 fascial layers hydrodissection and subsequent separation provide a nonthermal less traumatic cushioning between capsule and neurovascular bundle. Often this allows a greater margin of error for blunt dissection. ${ }^{10,11}$

During a partial nephrectomy, the ERBEJET 2 increases precision around vital structures due to sparing vessels during dissection and decreases blood loss, even without implementing ischemic techniques. ${ }^{6,8,9}$

Our article describes a new application of hydrodissection in laparoscopic transperitoneal bilateral ureterolysis for retroperitoneal fibrosis.

This procedure has never been described in the literature; we did it for the first time, and we think that it has many advantages so it will be useful to share it with other urologists who can benefit from this experience.

\section{Methods}

\section{Clinical presentation}

This diabetic patient presented to the ER for general status alteration and vomiting, lab tests revealed renal failure and hyperkalemia (serum creatinine $=10 \mathrm{mg} / \mathrm{dL}$ and $K=7 \mathrm{mg}$ / $\mathrm{dL}$ ), he had a history of chronic lumbar pain explored by $\mathrm{x}$-rays that were normal.

A KUB scan showed bilateral ureteropelvic dilatation over a retroperitoneal tissue thickening sheathing the aorta, inferior vena cava and the ureters in the lower lumbar area, a finding that can be considered as retroperitoneal fibrosis (Figure 1).

After stabilization, Double J stents were inserted bilaterally, the patient was given prednisone $40 \mathrm{mg}$ that was not tolerated because of his diabetes mellitus type II (D.M II), and so a laparoscopic transperitoneal bilateral ureterolysis using hydrodissection was programmed two months later after written informed consent has been provided by the patient.

\section{Hydrodissection material}

In this intervention, we used The ERBEJET 2 which is a water jet surgical device for the preparation and separation of tissue, suitable for preserving layering. The normal saline liquid used for dissection was warmed to $37^{\circ} \mathrm{C}$ temperature.

The rigid angled tip Hydro-Jet probe (ERBE $\left.{ }^{\mathrm{TM}}\right)$ and a suction traditional device tip were used for this procedure. The pressure was set at $600 \mathrm{psi}$ (41 bar) to allow for ureter dissection from the fibrosis with preservation of ureteral sheath and vessels with no use of electrosurgery on the ureters.

\section{Surgical technique}

Under general anesthesia, an esophageal probe was used to measure the patient's core temperature reliably and to record even rapid thermal changes. A Bair Hugger ${ }^{\mathrm{TM}}$ system was used, and a Foley catheter was inserted as well.

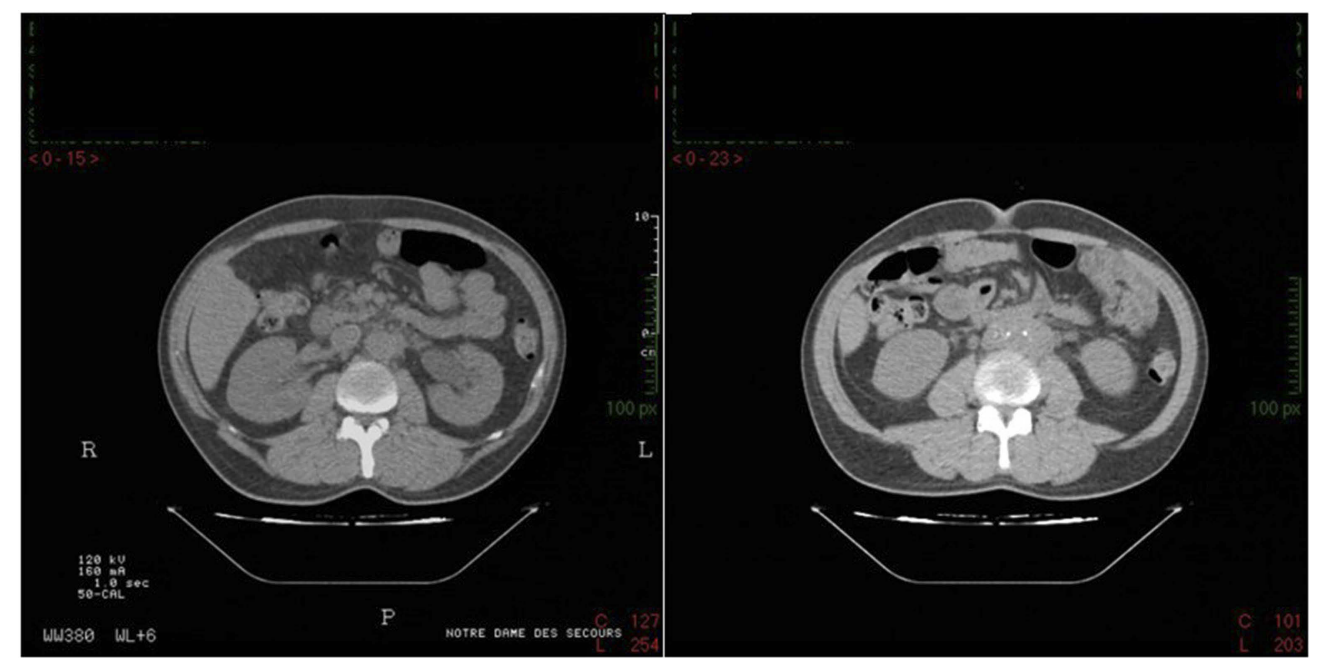

Figure I A kidney ureter bladder scan of the patient showing bilateral ureteropelvic dilatation over the retroperitoneal fibrosis sheathing the aorta, the inferior vena cava and the ureters in the lower lumbar area before (left image) and after (right image) double J stent insertion. 


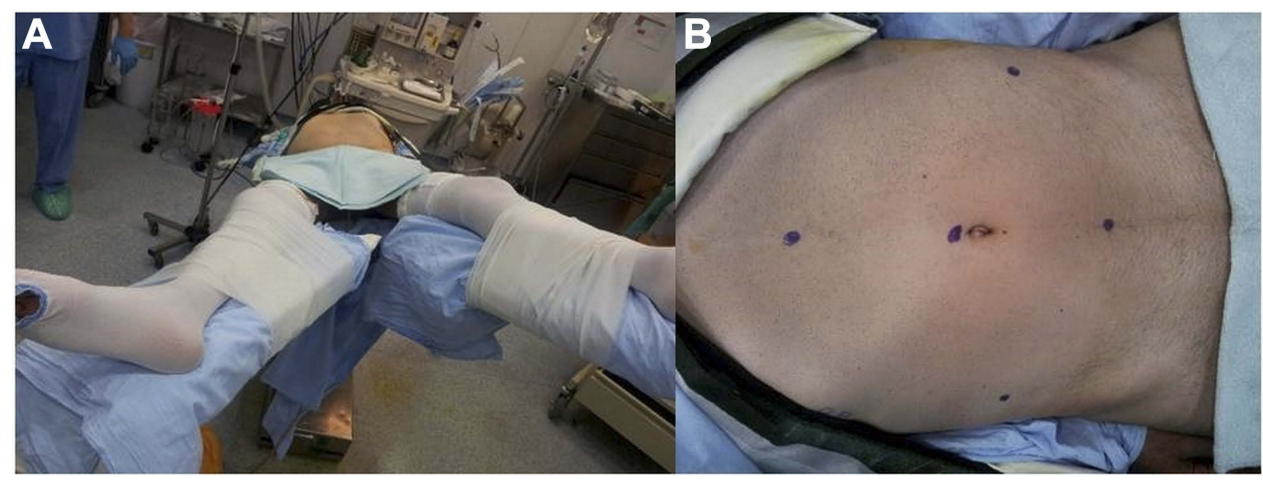

Figure 2 Patient surgical position (A) and trocars' points (B).

The patient was in the supine position. His legs were extended and spaced from each other, and the table was tilted to the right and to the left so that only five trocars can be used for both sides of ureterolysis without moving the patient (Figure 2A and B).

Pneumoperitoneum was achieved by insufflation of carbon dioxide using a VERESS needle through the umbilicus. A $10-\mathrm{mm}$ umbilical trocar was inserted into the peritoneal cavity permitting the introduction of a $10-\mathrm{mm}$ laparoscope. Two access ports were positioned medially to the bilateral anterior iliac spine, another was placed halfway between the umbilicus and the pubic symphysis and the last one was placed $3 \mathrm{~cm}$ under the xiphoid process (Figure 2A and B).

For the left side, the surgical table was tilted $60^{\circ}$ to the right to allow the bowel to drop away from the surgical field. Adhesions of the left paracolic area were dissected using electrocautery scissors. So was Toldt's fascia deeply till the psoas muscle. The descending colon was reflected medially and mobilized enough to reveal the area of fibrous tissue over the probable location of the ureter.

Identification of the ureter was difficult due to its encasement by the fibrotic tissue, this is why we identified the ureter in its proximal and distal segments (less fibrotic encasement): distally, at the level of its crossing over the iliac vessels and proximally near the left gonadal vein and medial to the lower pole of the left kidney.

After it was identified, the ureter was liberated from its fibrous plaque using hydrodissection (Figure 3), then it was mobilized in an area above the level of the plaque. Then, we dissected the ureter off the plaque all over its target using the rigid angled tip Hydro-Jet probe of ERBEJET 2 device with a continuous water stream pressure of 41 bar to allow the preservation of ureteral sheath and vessels with no use of electrosurgery (Figure 4).

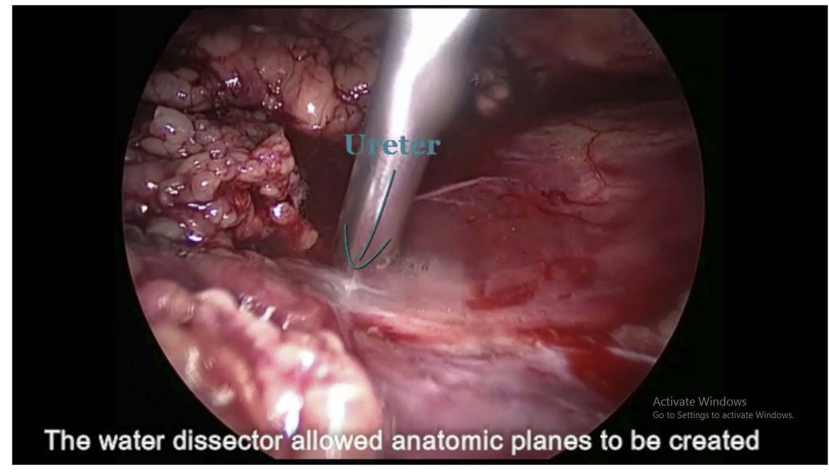

Figure 3 Identification and liberation of the ureter from its fibrous plaque using hydrodissection.

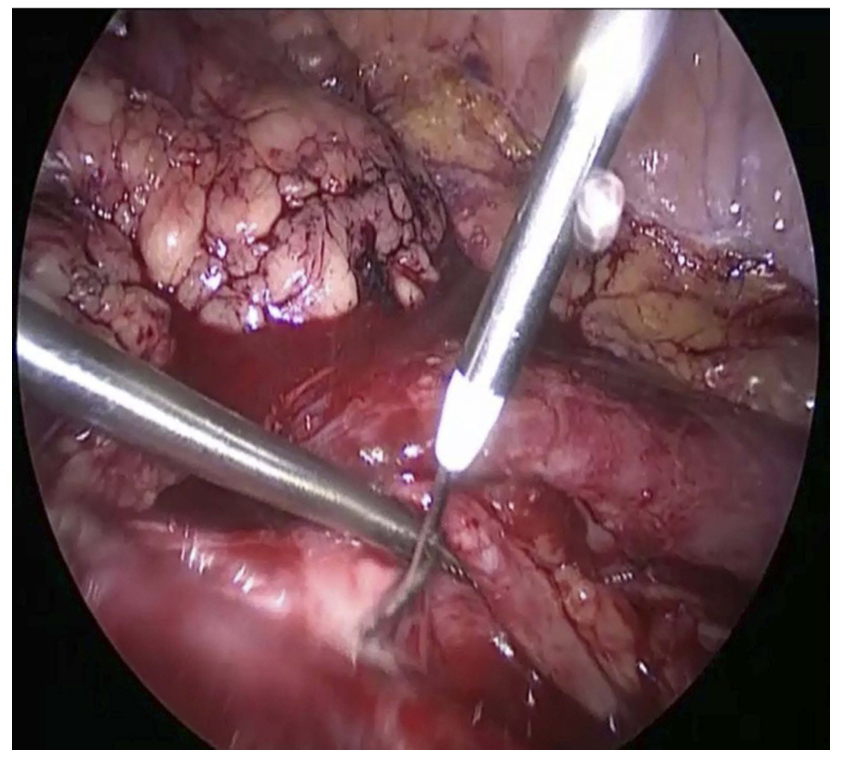

Figure 4 Dissection of the ureter of the plaque all over its target using the water jet (left side).

Continuous suction of the flushed normal saline by a traditional suction device tip was necessary during all the hydrodissection time. 


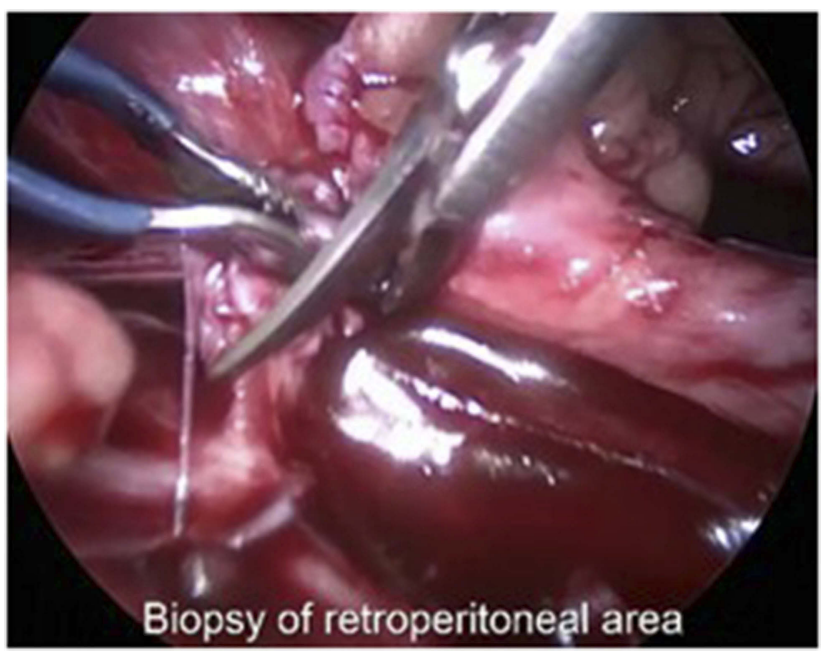

Figure 5 Biopsy from the fibrous tissue in the retroperitoneum.

After that, we took a biopsy from the fibrous tissue in the retroperitoneal area by a traditional method using laparoscopic scissors (Figure 5).

We ended with a completely released intact upper and mid ureter, which was lifted anteriorly to the left colon (Figure 6).

Finally, we closed Toldt's fascia with running sutures (Vicryl 3/0).

Moving to the right side, the same procedure was done after tilting the surgical table to the left: a dissection of Toldt's fascia was undergone as well and the right ureter was identified on the right lateral border of the inferior vena cava and medial to the lower pole of the right kidney. Once the right upper and mid ureter was isolated, it was also totally dissected using hydrojet with the same parameters (Figure 7).

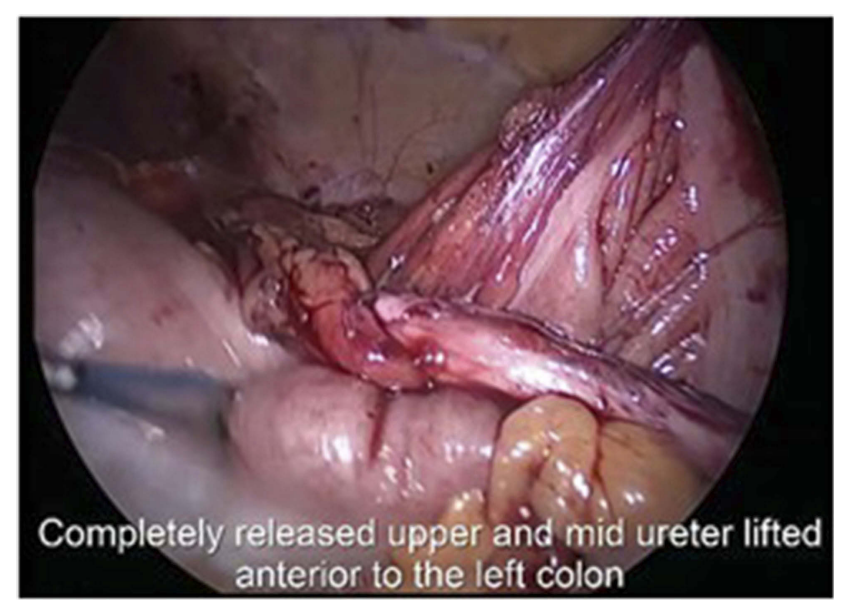

Figure 6 Completely released upper and mid ureter lifted anteriorly to the left colon.

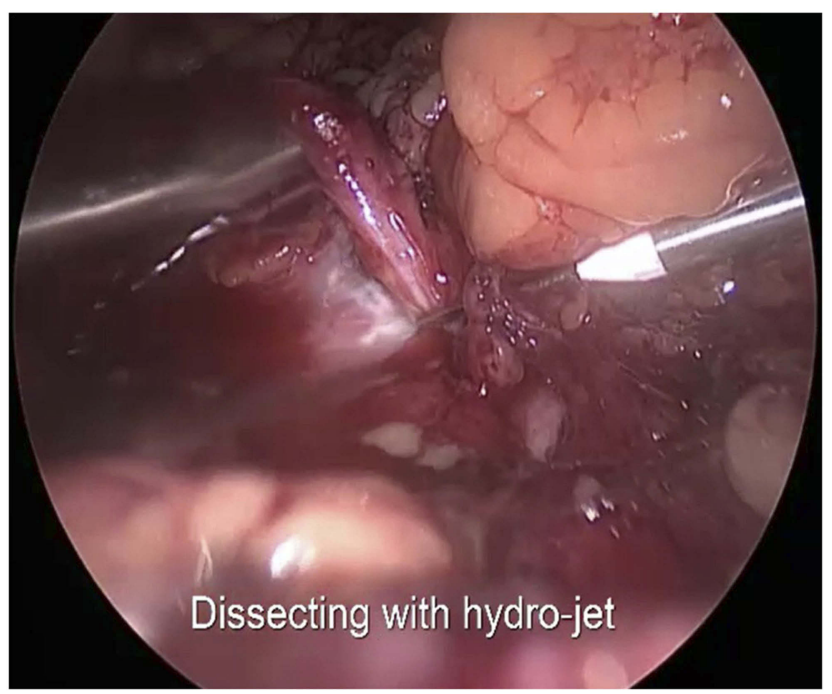

Figure 7 Hydrodissection of the ureter: less damaging (right side).

At this side, we decided to perform an omental wrapping around the whole hydrodissected ureter: A portion of the omentum that could be easily pulled and wrapped circumferentially around the ureter was used to isolate it from the retroperitoneum, and finally we transfixed the wrapped omentum with Vicryl 4-0 sutures.

\section{Results}

The whole operating time was 198 mins. No changes in the patient's temperature were recorded during the intervention. The blood loss was $50 \mathrm{cc}$ with no difference between the volumes of normal saline flushed and aspired during and at the end of the surgery. The post-operative lab tests were normal. The hospital stay was 3 days. The Double J stents were removed at 4 weeks postoperatively. Two years later, the patient was free of symptoms with normal serum creatinine level and no urinary tract obstructions.

\section{Discussion}

Hydrodissection of the ureters in retroperitoneal fibrosis is a new tool in the upper urinary tract using a laparoscopic approach. This new application was not described in the literature before.

It seems to simplify the ureterolysis and make it feasible and safe since it leads to higher selectivity compared with conventional techniques and spares blood vessels and nerves more effectively, which leads to lower blood loss.

No thermal damage occurs in the surrounding tissue, which is why a higher percentage of residual, functioning organ volume can be maintained and like this the ureter conserves its viability and its peristaltic function. 
Scientific studies attest to short operation times, as well as better and faster healing with significantly reduced length of hospitalization in laparoscopic hepatic surgery using hydrodissection. ${ }^{12,13}$ However, no studies were done concerning the ureterolysis in retroperitoneal fibrosis.

Ergonomic and light handpieces can make work less fatiguing. Highly user-friendly and intuitive operation and uniquely matched accessories can reduce the time spent in preparing the operation and training personnel.

In our case, we encountered a technical problem which was the need of continuous aspiration of the flushed normal saline since the water stream was filling the abdomen during the hydrodissection. This could be considered as an inconvenient of this technique since it could be timeconsuming even if it did not affect the surgical results.

Another pitfall of the device is the standardization of the pressure parameters. In our case, we chose to set the water jet pressure at around 40 bar; this pressure, according to the literature, is the double of the pressure used in partial nephrectomies and the quadruple of the pressure used in nerve sparing radical prostatectomies. ${ }^{9-11}$

We considered that we should increase the pressure to this level to be able to free the ureter from the fibrosis. However, it is still a random choice due to the lack of information in the literature.

Finally, we believe that hydrodissection of the ureters in retroperitoneal fibrosis seems to offer excellent midterm outcomes. However, comparative studies with other traditional techniques need to be done to assess the role of hydrodissection in ureterolysis for retroperitoneal fibrosis and elucidate its adequate application and all its disadvantages.

\section{Ethics}

Written informed consent has been provided by the patient to have the case details and any accompanying images published. Institutional approval was not required to publish the case details.

\section{Disclosure}

The authors report no conflicts of interest in this work.

\section{References}

1. Kaehler GF, Sold MG, Fischer K, Post S, Enderle M. Selective fluid cushion in the submucosal layer by water jet: advantage for endoscopic mucosal resection. Eur Surg Res. 2007;39(2):93-97. doi:10.1159/000099597

2. Kockerling F, Yildirim C, Rose J, Scheidbach H, Geers P. Total mesorectal excision with the water-jet-dissection. Technique and results. Tech Coloproctol. 2004;8(suppl 1):s217-s225. doi:10.1007/ s10151-004-0163-4

3. Meyer L, Uberruck T, Koch A, Gastinger I. Resection of the spleen using the water jet dissection technique. J Laparoendosc Adv Surg Tech A. 2004;14(5):321-324. doi:10.1089/lap.2004.14.321

4. Oertel J, Gaab MR, Knapp A, Essig H, Warzok R, Piek J. Water jet dissection in neurosurgery: experimental results in the porcine cadaveric brain. Neurosurgery. 2003;52(1):153-159, discussion 159.

5. Shekarriz B, Upadhyay J, Jewett MA. Nerve-sparing retroperitoneal lymphadenectomy using hydro-jet dissection: initial experience. J Endourol. 2004;18(3):273-276. doi:10.1089/089277904773582895

6. Shekarriz B. Hydro-jet technology in urologic surgery. Expert Rev Med Devices. 2005;2(3):287-291. doi:10.1586/17434440.2.3.287

7. Shekarriz H, Shekarriz B, Kujath P, et al. Hydro-jet-assisted laparoscopic cholecystectomy: a prospective randomized clinical study. Surgery. 2003;133(6):635-640. doi:10.1067/msy.2003.155

8. Shekarriz H, Shekarriz B, Upadhyay J, Burk C, Wood DP Jr, Bruch HP. Hydro-jet assisted laparoscopic partial nephrectomy: initial experience in a porcine model. $J$ Urol. 2000;163 (3):1005-1008. doi:10.1016/S0022-5347(05)67872-X

9. Moinzadeh A, Hasan W, Spaliviero M, et al. Water jet assisted laparoscopic partial nephrectomy without hilar clamping in the calfmodel. J Urol. 2005;174(1):317-321. doi:10.1097/01.ju.0000161587.95033.c9

10. Khurshid GA, Adam PE, Zubair BM, James PO. Hydrodissection for preservation of neurovascular bundle during robot-assisted radical prostatectomy. Can J Urol. 2008;15(2):4000-4003.

11. Patel MI, Spernat D, Lopez-Corona E. Hydrodissection of neurovascular bundles during open radical prostatectomy improves postoperative potency. J Urol. 2011;186(1):233-237. doi:10.1016/j.juro.2011.03.022

12. Rau HG, Duessel AP, Wurzbacher S. The use of water-jet dissection in open and laparoscopic liver resection. HPB (Oxford). 2008;10:275-280. doi:10.1080/13651820802167706

13. Vollmer CM, Dixon E, Sahajpal A, et al. Water-jet dissection for parenchymal division during hepatectomy. HPB (Oxford). 2006;8:377-385. doi:10.1080/13651820600839449.
Research and Reports in Urology

\section{Publish your work in this journal}

Research and Reports in Urology is an international, peer-reviewed, open access journal publishing original research, reports, editorials, reviews and commentaries on all aspects of adult and pediatric urology in the clinic and laboratory including the following topics: Pathology, pathophysiology of urological disease; Investigation and treatment of urological disease; Pharmacology of drugs used for the treatment of urological disease. The manuscript management system is completely online and includes a very quick and fair peer-review system, which is all easy to use. Visit http://www.dovepress.com/ testimonials.php to read real quotes from published authors. 